\title{
A clinicopathological study of gastric stump carcinoma following proximal gastrectomy
}

\author{
Shigekazu Ohyama ${ }^{1}$, Masanori Tokunaga ${ }^{1}$, Naoki Hiki $^{1}$, Tetsu Fukunaga ${ }^{1}$, Junko Fujisaki ${ }^{2}$, Yasuyuki Seto ${ }^{3}$, \\ and TOSHIHARU Yamaguchi ${ }^{1}$
}

${ }^{1}$ Department of Surgery, Cancer Institute Hospital of JFCR, 3-10-6 Ariake, Koto-ku, Tokyo 135-8550, Japan

${ }^{2}$ Department of Internal Medicine, Cancer Institute Hospital of JFCR, Tokyo, Japan

${ }^{3}$ Department of Gastrointestinal Surgery, The University of Tokyo, Graduate School of Medicine, Tokyo, Japan

\begin{abstract}
Background. We aimed to clarify the frequency and clinicopathological characteristics of gastric stump carcinoma following proximal gastrectomy.

Methods. Three-hundred and sixteen patients who had undergone curative proximal gastrectomy over a 21-year period from January 1984 through December 2004 were reviewed.

Results. Gastric stump carcinoma was observed in 17 patients $(5.4 \%)$. The time interval between the initial gastrectomy and the treatment of gastric stump cancer was within 5 years in 3 patients, within 5-10 years in 8, and after 10 years in 6. Treatment included endoscopic resection $(n=4)$, completion total gastrectomy of the remnant $\operatorname{stomach}(n=11)$, pancreatoduodenectomy $(n=1)$, and nonsurgical resection $(n=1)$. Pathologically, 9 carcinomas were differentiated and 8 were undifferentiated. In a review of reconstruction methods associated with disease stage, stage $I$ was found in 6 of the 7 patients with esophagogastrostomy or short-segment jejunal interposition. On the other hand, stage I was found in only 3, but stage II-IV was found in 7 of the 10 patients with reconstruction by double-tract or long-segment jejunal interposition; thus, the tumor was more likely to be detected at an advanced stage after long-segment interposition $(P=0.049)$.

Conclusion. Gastric stump carcinoma following proximal gastrectomy occurred at a high frequency of $5.4 \%$ of initial resections. It is necessary to select a reconstruction method that facilitates postoperative endoscopic examination, as well as to follow up the patients after proximal gastrectomy in the long term for the early detection and early treatment of gastric stump carcinoma.
\end{abstract}

Key words Gastric stump carcinoma - Proximal gastrectomy · Jejunal interposition

Offprint requests to: $\mathrm{S}$. Ohyama

Received: April 24, 2008 / Accepted: February 4, 2009

\section{Introduction}

Proximal gastrectomy has been widely accepted as function-preserving surgery for early gastric cancer located in the cardiac part of the stomach [1-3]. However, the procedure has two important problems; one is the postoperative quality of life (QOL) involving reflux esophagitis $[4,5]$; the other is carcinoma arising in the gastric stump. To date, only a few studies have been published on gastric stump carcinoma in patients who have undergone proximal gastrectomy [6-8], although many studies have revealed the clinicopathological findings of gastric stump cancer after distal gastrectomy [9-14]. We have experienced 17 cases of gastric stump carcinoma arising after proximal gastrectomy. This study aimed to reveal the clinicopathological features of gastric stump cancer following proximal gastrectomy.

\section{Patients and methods}

\section{Proximal gastrectomy}

We reviewed 316 curative proximal gastrectomies among 6151 patients who had undergone stomach resection between January 1984 and December 2004 at the Cancer Institute Hospital, Tokyo, Japan. Proximal gastrectomy was indicated for early gastric cancer located in the cardiac portion of the stomach, and exceptionally for bleeding gastric ulcer. The reconstruction method after proximal gastrectomy was changed during the study period. From 1984 through 1994, a double-tract method or long-segment jejunal interposition (long interposition) was adopted after resection of the upper half of the stomach. In these reconstructions, the distance between the esophagojejunal anastomosis and the jejunogastrostomy was 30 to $40 \mathrm{~cm}$, and pyloroplasty was required. After 1995, as the resection range had been changed to the proximal-third or proximal 
quarter of the stomach, short-segment jejunal interposition (short interposition), in which the length of the esophagogastric anastomosis or interposed jejunum was within $15 \mathrm{~cm}$, was then possible. With this method, the hepatic branch, pyloric branch, and celiac branch of the vagus nerve were preserved, and pyloroplasty was not needed. During the entire study period, the double tract method was adopted in 78 patients, long interposition in 142, esophagogastrostomy in 73 , and short interposition in 23. Figure 1 illustrates the reconstruction methods following proximal gastrectomy.

\section{Definition of gastric stump carcinoma}

Gastric stump carcinoma was defined as: (1) carcinoma detected more than 10 years after the initial surgery for either malignant or benign disease; or (2) carcinoma detected within 10 years that was likely to have occurred independently of the initial lesion [15]. Cancers that developed at the anastomotic site or on the suture line within 10 years after the initial surgery for a malignant lesion, and those that developed at the anastomotic site or on the suture line with a positive resection margin after the initial surgery for a malignant lesion were excluded from the study.

\section{Postoperative follow-up}

Postoperative follow-up after the double-tract method or long interposition was conducted annually by postoperative fluoroscopy and computed tomography (CT) scan. Endoscopy was also carried out every year, but observation by this method was insufficient in many patients with these reconstruction methods.

After esophagogastrostomy or short interposition, upper endoscopy as well as CT scan was conducted annually. Follow-up after 5 years was performed at the patient's request at our institute or at a nearby hospital.

In the present study, we reviewed gastric stump cancers after proximal gastrectomy in terms of clinicopathological features at the initial surgery, reconstruction methods, time to the treatment of gastric stump cancer, and pathological findings of the gastric stump carcinomas. The terms used here are based on the Japanese classification of gastric carcinoma (second English edition) [16]. Statistical analysis was performed by using Fisher's exact test.

\section{Results}

Gastric stump carcinoma was found in 17 (5.4\%) of the 316 patients who had undergone curative proximal gastrectomies. Table 1 and Table 2 show the clinicopatho-
Table 1. Clinicopathologic characteristics of patients at initial surgery

\begin{tabular}{|c|c|}
\hline Number of patients & 17 \\
\hline \multicolumn{2}{|l|}{ Sex } \\
\hline Male & 15 \\
\hline Female & 2 \\
\hline \multicolumn{2}{|l|}{ Age (years) } \\
\hline Average & 59.3 \\
\hline Range & $39-71$ \\
\hline \multicolumn{2}{|l|}{ Reconstruction } \\
\hline Double-tract & 3 \\
\hline Interposition $^{\mathrm{a}}$ & 7 \\
\hline EG & 3 \\
\hline Short interposition ${ }^{\mathrm{b}}$ & 4 \\
\hline \multicolumn{2}{|l|}{ Macroscopic type } \\
\hline 0-IIa & 3 \\
\hline 0-IIc & 12 \\
\hline \multicolumn{2}{|l|}{ Histology } \\
\hline Differentiated & 13 \\
\hline Undifferentiated & 2 \\
\hline \multicolumn{2}{|l|}{ Tumor depth } \\
\hline $\mathrm{m}$ & 7 \\
\hline sm & 7 \\
\hline $\mathrm{mp}$ & 1 \\
\hline \multicolumn{2}{|c|}{ Degree of lymph node metastasis } \\
\hline n0 & 12 \\
\hline $\mathrm{n} 1$ & 3 \\
\hline n2 & 0 \\
\hline \multicolumn{2}{|l|}{ Stage } \\
\hline I & 14 \\
\hline II & 1 \\
\hline
\end{tabular}

EG, esophagogastrostomy; Interposition, jejunal interposition

${ }^{\text {a }}$ Long-segment jejunal interposition of 30- to $40-\mathrm{cm}$ length

${ }^{\mathrm{b}}$ Short-segment jejunal interposition of 5- to 15 -cm length

logical characteristics of the 17 patients. At the initial surgery, the lesions in 2 patients were benign; one was a bleeding gastric ulcer and the other was an adenoma, confirmed on the postoperative pathological examination, but which had been preoperatively diagnosed as carcinoma. Of the 15 malignant lesions, all but 1 was at an early stage; pathologically 13 of them were differentiated and the remaining 2 were undifferentiated tumors. After the proximal gastrectomy, 3 patients had reconstruction by esophagogastrostomy, 3 by the double-tract method, and 11 by jejunal interposition, including 4 with short interposition. The time to treatment of the gastric stump carcinoma was within 5 years in 3 patients, within $5-10$ years in 8 , and after 10 years in 6. Treatment of the gastric stump carcinoma included endoscopic resection in 4 patients, total completion gastrectomy of the gastric remnant in 11 , and pancreatoduodenectomy in 1 . One patient did not receive surgical treatment. Pathologically, 9 carcinomas were differentiated and 8 were undifferentiated. When examined in relation to the initial disease, differentiated tumor in the gastric stump carcinoma was found in 9 of the 13 patients with differentiated histology in the initial disease. The 2 undifferentiated initial lesions had, again, 

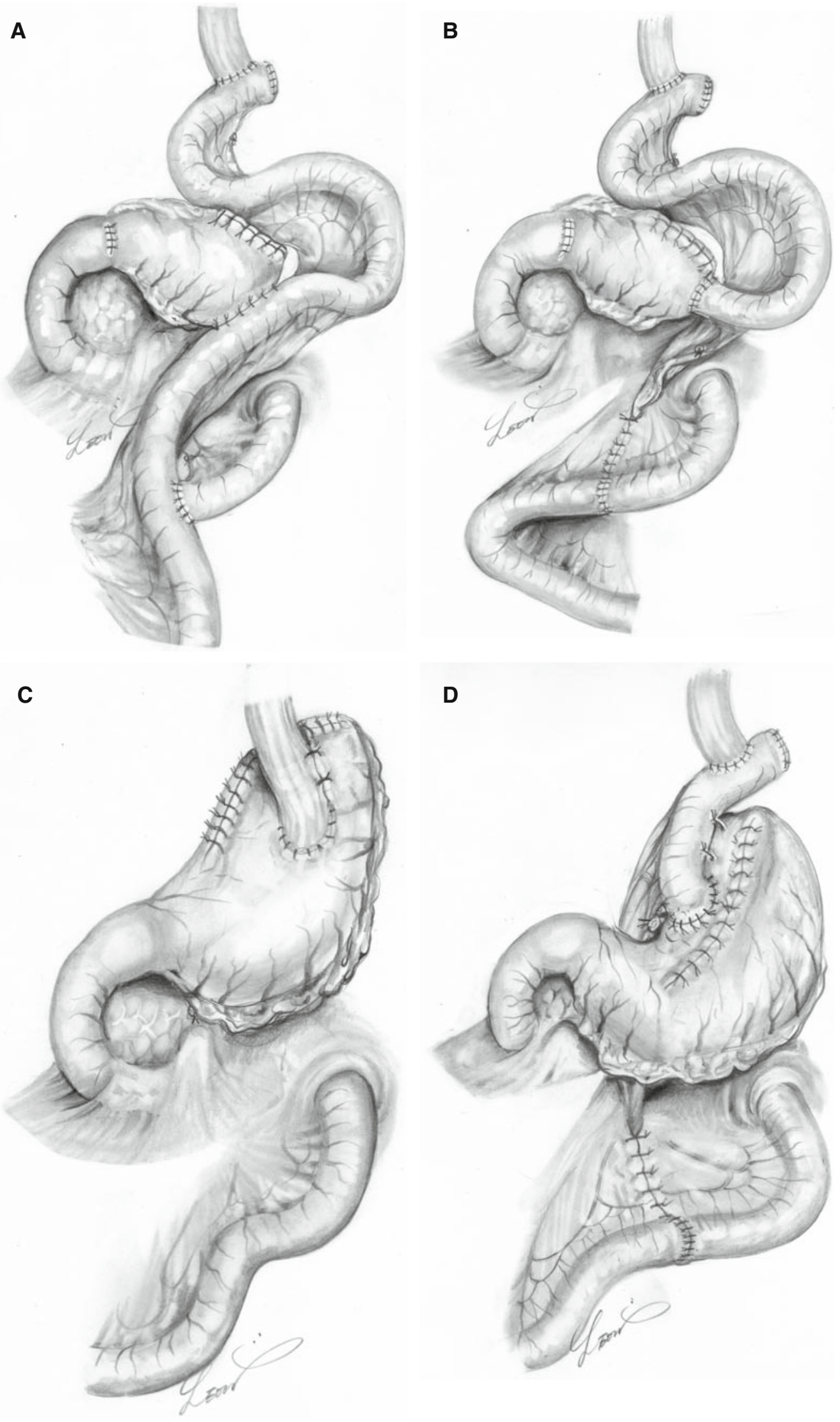

Fig. 1A-D. Reconstruction methods following proximal gastrectomy. A Double-tract reconstruction; B long-segment jejunal interposition; $\mathbf{C}$ esophagogastrostomy; $\mathbf{D}$ short-segment jejunal interposition 


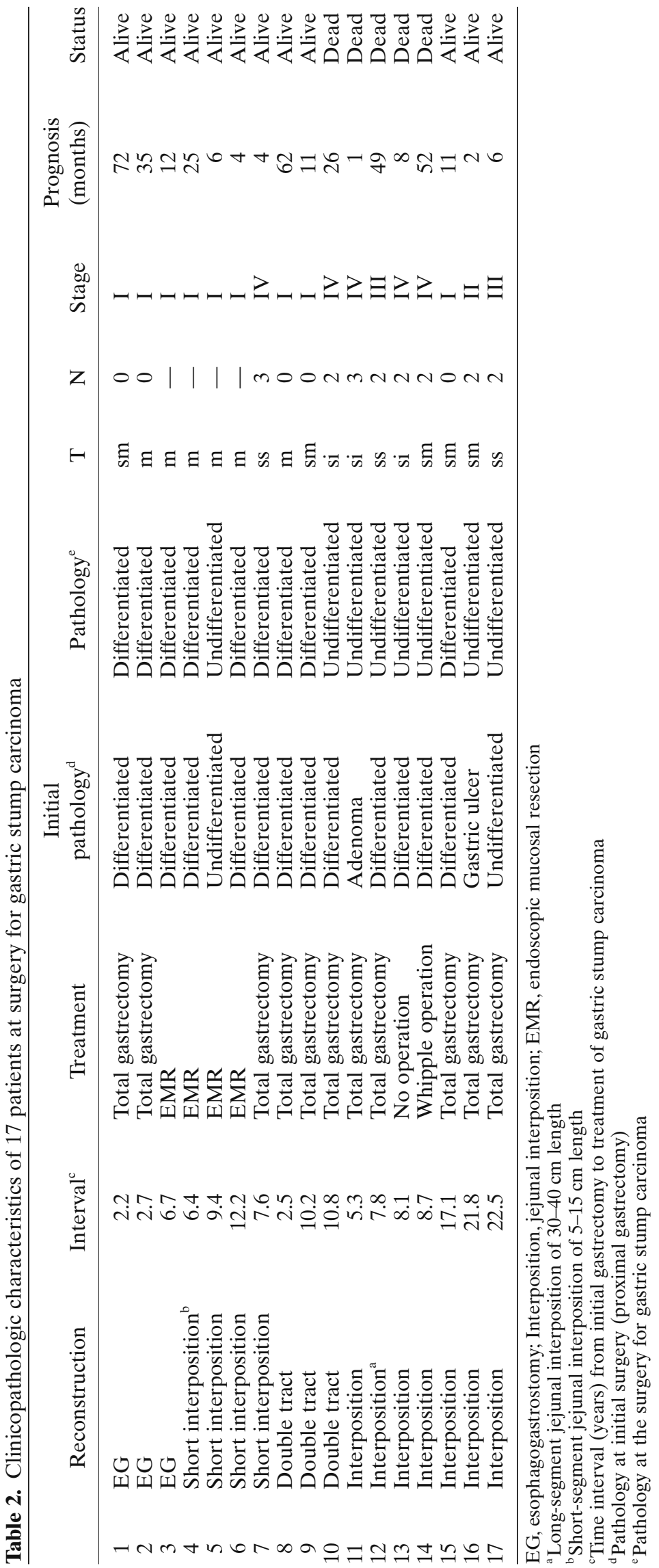


undifferentiated pathology in the gastric stump carcinomas (Table 3). With regard to the stage of the gastric stump cancers, stage I disease was found in 9 patients, stage II in 1 , stage III in 2 , and stage IV in 5 . When we reviewed the reconstruction methods associated with cancer stage, gastric stump carcinoma was detected at stage I in all 3 patients with reconstruction by esophagogastrostomy and 3 of the 4 with reconstruction by short interposition. On the other hand, stage I was found in only 3 of the 10 patients with a long-interposition or double-tract reconstruction; in contrast, these reconstruction methods were associated with stage II, III, or IV disease in 7 of the 10 patients with such reconstruction. Thus, gastric stump carcinomas were more likely to be detected at an advanced stage after a doubletract or long jejunal interposition reconstruction method (Table $4 ; P=0.049$ ).

\section{Discussion}

Many studies have shown that gastric stump cancer develops after distal gastrectomy, particularly after Billroth II reconstruction, at frequencies of $0.4 \%-2.5 \%$, with an increased incidence 15 years after surgery [1720]. Reflux of duodenal fluid such as bile or pancreatic juice was reported to be associated with the development of gastric carcinoma after distal gastrectomy [21-26].

On the other hand, very few studies have been conducted on gastric stump carcinoma after proximal gastrectomy. Igami et al. [8] reported that gastric stump cancer was found in $6(5 \%)$ of 120 patients who received

Table 3. Comparison of pathological types of initial lesion and gastric stump carcinoma

$\begin{array}{ll}\text { Initial lesion } & \\ \text { Differentiated } & 2 \\ \text { Undifferentiated } & 1\end{array}$

proximal gastrectomy over a 27-year period from 1971 to 1997. Kaminishi et al. [7] showed 26 cases of gastric stump cancer following proximal gastrectomy, but the incidence was not studied because the total case number was unclear. In our review, gastric stump carcinoma was observed at a frequency of $5.4 \%$ in patients with curative proximal gastrectomies. It is presumed that gastric stump cancer develops at a higher frequency after proximal gastrectomy than after distal gastrectomy.

As for the cause of gastric stump carcinoma after proximal gastrectomy, the following reasons have been advanced: (1) the distal part of the stomach is originally a high-incidence area for gastric carcinoma; (2) postoperative hypergastrinemia promotes carcinogenesis (after resection of the fundic gland region in proximal gastrectomy, anacidity occurs and parietal cells are decreased in quantity and function [27-29]; and (3) expression of the trefoil factor family 1 [30] (TFF1; pS2), which inhibits carcinogenesis, is decreased after resection of the fundic gland region. It was reported that TFF1 knockout mice developed adenomas and carcinomas in the stomach. It is thought that such factors are contributors to the high risk of gastric carcinoma after proximal gastrectomy [31-33].

Concerning the pathological type at the initial resection, differentiated carcinoma was more frequently observed in our study. In this regard, the possibility of selection bias cannot be denied; that is, proximal gastrectomy was more likely to be performed for differentiated carcinoma than for undifferentiated carcinoma, because the spread of undifferentiated carcinoma is unclear. Kaminishi et al. [7] reported that differentiated carcinoma was more frequent, being observed in 11 of 15 pathologically confirmed gastric stump cancers following proximal gastrectomy. In our review, the numbers of differentiated and undifferentiated tumors in the gastric stump carcinomas were almost the same.. Because there have been only a few studies published on the pathological type of gastric stump carcinoma to date, its characteristics still remain unclear.

Concerning the time interval to the occurrence of gastric stump cancer, Kaminishi et al. [7] reported that occurrence within 10 years after the initial surgery was the most common period, with 11 patients (42\%)

Table 4. Reconstruction methods in relation to cancer stage

\begin{tabular}{|c|c|c|c|c|}
\hline & \multirow{2}{*}{$\frac{\text { Early gastric cancer }}{\text { Stage I }}$} & \multicolumn{3}{|c|}{ Advanced gastric cancer } \\
\hline & & Stage II & Stage III & Stage IV \\
\hline Double tract & 2 & & & 1 \\
\hline Jejunal interposition $^{\mathrm{a}}$ & 1 & 1 & 2 & 3 \\
\hline Esophagogastrostomy & 3 & & & \\
\hline Short jejunal interposition ${ }^{\mathrm{b}}$ & 3 & & & 1 \\
\hline
\end{tabular}


showing occurrence within 5 years, $9(35 \%)$ showing occurrence between 5 and 10 years, and $6(23 \%)$ showing occurrence after 10 years. As we also found that most patients $(n=11)$ developed carcinomas within 10 years after surgery, it is necessary to conduct careful followup within 10 years. Also, as some gastric stump carcinomas were detected more than 10 years after the initial surgery, long-term follow-up even after 10 years is essential.

Reconstruction following proximal gastrectomy was conventionally focused on preventing postoperative reflux esophagitis. Thus, a double-tract method or long interposition, in which the esophagojejunal anastomosis was located $30 \mathrm{~cm}$ or more distant from the jejunogastrostomy, was frequently adopted. However, in patients who had had these reconstruction methods there were some difficulties in conducting endoscopic examinations of the remnant stomach. In recent years, with the increasing incidence of early gastric cancer located in the upper third of the stomach, the procedure of proximal gastrectomy has been changed, with conservation of a large part of the stomach, preservation of the vagus nerves, or the creation of a jejunal pouch. Igami et al. [8] employed esophagogastrostomy in all their patients, while Katai et al. [2] recommended the use of short interposition. Kikuchi et al. [34] also reported the efficacy of jejunal pouch interposition in an attempt to increase food intake. Still, it has remained controversial as to which reconstruction method should be used following gastric resection, and different surgical procedures have been employed.

To detect gastric stump cancer at an early stage, it is essential to conduct endoscopic follow-up of the remnant stomach. When we examined the reconstruction methods associated with disease stage, six of the seven patients who had received esophagogastrostomy or short interposition were found to have stage I disease; four of these patients were treated with endoscopic resection. Among the patients reconstructed by conventional procedures such as long interposition or the double-tract method, the incidence of stage I disease was only $30 \%$, whereas stage IV disease was found in four of these patients $(40 \%)$. After a double-tract or long-interposition reconstruction, gastric stump carcinoma was more likely to be detected at an advanced stage, even including an unresectable tumor. This indicated that a reconstruction method that facilitates postoperative endoscopic observation of the remnant stomach is useful for the early detection and early treatment of gastric stump carcinoma after proximal gastrectomy.

Proximal gastrectomy will require further efforts to improve patients' postoperative QOL. The present study clarified that there was a high frequency of gastric stump carcinoma following proximal gastrectomy. It is necessary to select an appropriate reconstruction method that facilitates postoperative endoscopic examination. This will contribute to the early detection and early treatment of gastric stump carcinomas following proximal gastrectomy in the future.

Acknowledgments All figures were drawn by special medical illustrator Mr. Leon Sakuma.

\section{References}

1. Harrison LE, Karpeh MS, Brennan MF. Total gastrectomy is not necessary for proximal gastric cancer. Surgery 1998;123:12730.

2. Katai H, Sano T, Fukagawa T, Shinohara H, Sasako M. Prospective study of proximal gastrectomy for early gastric cancer in the upper third of the stomach. Br J Surg 2003;90:850-3.

3. Iwata T, Kurita N, Ikemoto T, Nishioka M, Andoh T, Shimada M. Evaluation of reconstruction after proximal gastrectomy: prospective comparative study of jejunal interposition and jejunal pouch interposition. Hepatogastroenterology 2006;53:301-3.

4. Takeshita K, Saito N, Saeki I, Honda T, Tani M, Kando F, et al. Proximal gastrectomy and jejunal pouch interposition for the treatment of early cancer in the upper third of the stomach: surgical techniques and evaluation of postoperative function. Surgery 1997;121:278-86.

5. Hoshikawa T, Denno R, Ura H, Yamaguchi K, Hirata K. Proximal gastrectomy and jejunal pouch interposition: evaluation of postoperative symptoms and gastrointestinal hormone secretion. Oncol Rep 2001;8:1293-9.

6. Nozaki I, Kurita A, Nasu J, Kubo Y, Aogi K, Tanada M, et al. Higher incidence of gastric remnant cancer after proximal than distal gastrectomy. Hepatogastroenterology 2007;54: 1604-8.

7. Kaminishi N, Shimizu N, Hiki N, Nomura Y, Nozaki K, Imamura $\mathrm{K}$, et al. Clinicopathological features of gastric remnant cancer after corpofundectomy for gastric cancer (In Japanese). Stomach and Intestine 2004;39:1049-57.

8. Igami G, Isotani M, Harada T, Kaneoka Y, Akutagawa A, Sugawara $\mathrm{G}$, et al. Remnant stomach cancer after proximal gastrectomy: a review of 26 cases in the Japanese literature. Jpn J Gastroenterol Surg 2002;35:357-61.

9. Newman E, Brennan MF, Hochwald SN, Harrison LE, Karpeh MS Jr. Gastric remnant carcinoma: just another proximal gastric cancer or a unique entity? Am J Surg 1997;173:292-7.

10. Kaneko K, Kondo H, Saito D, Shirao K, Yamaguchi H, Yokota $\mathrm{T}$, et al. Early gastric stump cancer following distal gastrectomy. Gut 1998;43:342-4.

11. Ikeda Y, Saku M, Kishihara F, Maehara Y. Effective follow-up for recurrence or a second primary cancer in patients with early gastric cancer. Br J Surg 2005;92:235-9.

12. Sinning C, Schaefer N, Standop J, Hirner A, Wolff M. Gastric stump carcinoma - epidemiology and current concepts in pathogenesis and treatment. Eur J Surg Oncol 2007;33:133-9.

13. Ishikawa M, Kitayama J, Kaizaki S, Nakayama H, Ishigami H, Fujii S, et al. Prospective randomized trial comparing Billroth I and Roux-en-Y procedures after distal gastrectomy for gastric carcinoma. World J Surg 2005;29:1415-20; discussion 1421.

14. Ohashi M, Katai H, Fukagawa T, Gotoda T, Sano T, Sasako M. Cancer of the gastric stump following distal gastrectomy for cancer. Br J Surg 2007;94:92-5.

15. Kaminishi M, Shimizu N, Shiomoyama S, Yamaguchi H, Ogawa T, Sakai S, et al. Etiology of gastric remnant cancer with special reference to the effects of denervation of the gastric mucosa. Cancer 1995;75:1490-6. 
16. Japanese Gastric Cancer Association. Japanese classification of gastric carcinoma - 2nd English edition - Gastric Cancer 1998;1:10-24.

17. Tokudome S, Kono S, Ikeda M, Kuratsune M, Sano C, Inokuchi $\mathrm{K}$, et al. A prospective study on primary gastric stump cancer following partial gastrectomy for benign gastroduodenal diseases. Cancer Res 1984;44:2208-12.

18. Asano A, Mizuno S, Sasaki R, Aoki K, Yokoyama H, Yokoyama Y. The long-term prognosis of patients gastrectomized for benign gastroduodenal diseases. Jpn J Cancer Res 1987;78:337-48.

19. Inokuchi K, Tokudome S, Ikeda M, Kuratsune M, Ichimiya H, Kaibara N, et al. Mortality from carcinoma after partial gastrectomy. Gann 1984;75:588-94.

20. Viste A, Bjornestad E, Opheim P, Skarstein A, Thunold J, Hartveit F, et al. Risk of carcinoma following gastric operations for benign disease. A historical cohort study of 3470 patients. Lancet 1986;2:502-5.

21. Fisher SG, Davis F, Nelson R, Weber L, Goldberg J, Haenszel W. A cohort study of stomach cancer risk in men after gastric surgery for benign disease. J Natl Cancer Inst 1993;85:130310.

22. Toftgaard C. Gastric cancer after peptic ulcer surgery. A historic prospective cohort investigation. Ann Surg 1989;210:159-64.

23. Lundegardh G, Adami HO, Helmick C, Zack M, Meirik O. Stomach cancer after partial gastrectomy for benign ulcer disease. N Engl J Med 1988;319:195-200.

24. Miwa K, Hattori T, Miyazaki I. Duodenogastric reflux and foregut carcinogenesis. Cancer 1995;75:1426-32.

25. Kondo K, Kojima H, Akiyama S, Ito K, Takagi H. Pathogenesis of adenocarcinoma induced by gastrojejunostomy in Wistar rats: role of duodenogastric reflux. Carcinogenesis 1995;16:1747-51.
26. Byrne JP, Attwood SE. Duodenogastric reflux and cancer. Hepatogastroenterology 1999;46:74-85.

27. Langhans N, Rindi G, Chiu M, Rehfeld JF, Ardman B, Beinborn $\mathrm{M}$, et al. Abnormal gastric histology and decreased acid production in cholecystokinin-B/gastrin receptor-deficient mice. Gastroenterology 1997;112:280-6.

28. Wang TC, Dangler CA, Chen D, Goldenring JR, Koh T, Raychowdhury R, et al. Synergistic interaction between hypergastrinemia and Helicobacter infection in a mouse model of gastric cancer. Gastroenterology 2000;118:36-47.

29. Koh TJ, Goldenring JR, Ito S, Mashimo H, Kopin AS, Varro A, et al. Gastrin deficiency results in altered gastric differentiation and decreased colonic proliferation in mice. Gastroenterology 1997;113:1015-25.

30. Wright NA. Trefoil peptides and the gut. Gut 1993;34:577-9.

31. Hippo Y, Taniguchi H, Tsutsumi S, Machida N, Chong JM, Fukayama M, et al. Global gene expression analysis of gastric cancer by oligonucleotide microarrays. Cancer Res 2002;62: 233-40.

32. Taupin D, Pedersen J, Familari M, Cook G, Yeomans N, Giraud AS. Augmented intestinal trefoil factor (TFF3) and loss of pS2 (TFF1) expression precedes metaplastic differentiation of gastric epithelium. Lab Invest 2001;81:397-408.

33. Lefebvre O, Chenard MP, Masson R, Linares J, Dierich A, LeMeur M, et al. Gastric mucosa abnormalities and tumorigenesis in mice lacking the pS2 trefoil protein. Science 1996; 274:259-62.

34. Kikuchi S, Nemoto Y, Katada N, Sakuramoto S, Kobayashi N, Shimao H, et al. Results of follow-up endoscopy in patients who underwent proximal gastrectomy with jejunal interposition for gastric cancer. Hepatogastroenterology 2007;54:304-7. 